\title{
Phenotypic Characterization, Antimicrobial Susceptibility Patterns Profile and Risk Factors of Escherichia Colio157:H7 Isolated from Cattle Meat at Jimma Ethiopia
}

\author{
Eshetu Shumi $^{1,}$, , Tadele Tolosa ${ }^{1}$, Mukarim Abdurahaman ${ }^{1}$, Abebe Olani $^{2}$, Matios Lekew ${ }^{2}$, \\ Diriba Taddese ${ }^{1}$
}

${ }^{1}$ Jimma University College of Agriculture and Veterinary Medicine, Jimma, Oromia, Ethiopia

${ }^{2}$ National Animal Health Diagnostic and Investigation Center, Sebata, Oromia, Ethiopia

Email address:

eshetu.shumi@yahoo.com (E. Shumi), tadeletolosa@yahoo.com (T. Tolosa), mukevet@yahoo.com (M. Abdurahaman), anaol@gmail.com (A. Olani), Matioslekew@gmail.com (M. Lekew),dirrooballee@gmail.com (D. Taddese)

${ }^{*}$ Corresponding author

\section{To cite this article:}

Eshetu Shumi, Tadele Tolosa, Mukarim Abdurahaman, Abebe Olani, Matios Lekew, Diriba Taddese. Phenotypic Characterization, Antimicrobial Susceptibility Patterns Profile and Risk Factors of Escherichia Colio157:H7 Isolated from Cattle Meat at Jimma Ethiopia. American Journal of Bioscience and Bioengineering. Vol. 9, No. 2, 2021, pp. 40-48. doi: 10.11648/j.bio.20210902.12

Received: December 31, 2020; Accepted: March 25, 2021; Published: April 23, 2021

\begin{abstract}
Escherichia coli $\mathrm{O} 157: \mathrm{H} 7$ is considered the most prevalent food borne pathogen that has gained increasing attention worldwide in recent years. A cross sectional study was undertaken from January 2018 to September 2018 on carcass swab and utensil at slaughter house as well as meat and utensil obtained from butcher shop at Jimma town to isolate identify and assess association risk factor of Escherichia coli $\mathrm{O} 157: \mathrm{H} 7$ and its antimicrobial susceptibility patterns. From total of 288 samples 51 of them were biochemically positive for Escherichia coli. From those isolated by biochemical tests four of them were found to be positive for Escherichia coli O157:H7 strain as confirmed by Biolog incubation manual. Among the samples (144) from slaughter house examined 3 of the E. coli O157:H7 isolates were found to be positive for E. coli O157:H7. Two of the isolates were identified from carcass and one from hanging up wire at slaughter house while the rest one was from meat at butcher shop. The antimicrobial susceptibility investigation of $4 \mathrm{E}$. coli O157:H7 isolates using 12 commonly marketable antimicrobial discs revealed that the isolates were susceptible to eight antimicrobials from $75 \%$ to $100 \%$. Results of the present study indicated that an overall resistance of $100 \%, 75 \%, 50 \%$ and $25 \%$ was observed to Ampicillin, Neomycin, Cefoxitin and Oxytetracycline respectively. Multi drug resistance to three drugs was detected in $1(25 \%)$ of the isolates. This study indicates the possible risk of E. coli O157:H7 particularly for the consumers who have the habit of eating raw or undercooked meat. These findings stress the need for implementation of E. coli O157:H7 prevention and control strategies from slaughter house to butcher shop and consumption of meat.
\end{abstract}

Keywords: Abattoir, Antimicrobial, Butcher Shop, Escherichia Coli O157:H7, Isolation, Jimma, Raw Meat

\section{Introduction}

Food born disease is one of the vital issues in the in the $21^{\text {st }}$ century all over the world. Reports have indicated that, massive number of people suffers from food borne disease worldwide due to infected food and water utilization annually [22].

This indicates that microbial food borne illness still left over's a global concern despite the general scientific advancement and technological developments achieved [26].

Several epidemiological reports have concerned food of animal origin as the major vehicles associated with illness caused by food borne pathogens such as Escherichia coli, salmonella, shigellaand cambylobacter for human infection and this problem is highly aggravated in the developing world [26].

Among the pathogens Escherichia coli O157:H7 are major food borne pathogens that have been the center of food safety research in developing countries such as Ethiopia. Human infection with E. coli O157:H7 have been mostly associated with the consumption of contaminated and improperly cooked minced beef and unpasteurized cow milk. Butcher 
shop and restaurants are frequently incriminated as a sourse of E. coli O157:H7 for human infections [5, 12].

Cattle are the primary reservoirs of E. coli O157:H7 and ground beef and beef products are identified as major sourse of food borne transmission. Carcass contamination occurs through skin-to-carcass or fecal-to-carcass transfer of the pathogen during slaughter process at processing plants; and this is the major risk factor for human infection [32].

Many studies on the microbiological hygiene of cattle at slaughter have shown that hide contamination is strongly correlated with carcass contamination, which is likely the result of cross contamination, during processing [34].

Consumption of contaminated and/or uncooked meat poses the risk of acquiring food borne E. coli strain causing a serious public health concern. These bacterial strains easily harbor antibiotic resistance gene from one another. These is because gene encoding AMR determinants that are carried on mobile genetic elements such as plasmid and transposons of some bacterial strain could be transferred to other bacterial strains during contact, causing a treat to cure acute infection in man and animals [11].

Antibiotic use in VTEC infections is controversial because of the potential to increase production and secretion of Shiga toxins [33]. However, increase in antibiotic resistance has been noted over the last 20 years. Outbreak of E. coli O157:H7 has been reported in different parts of the world [29].

Recently published reports indicated that the E. coli strains isolated from contaminated meat and meat products have became resistant to commonly used antibiotics. This is mainly due to injudicious usage of antibiotics in both humans and animals. The wide spread and imprudent use of antibiotics in food animals is through to be accountable for the emergence and wider spreading of antimicrobial resistant (AMR) bacterial in humans [22].

In humans, positive selections for drug resistant bacteria have also been reported in the normal microfilora of exposed individuals or populations. This indicates that antibiotic resistance can be developed in both commensal and pathogenic bacterial strains and can even be transferred to other bacterial strains, including other pathogenic and environmental bacteria [19].

\subsection{Problem of Statement}

In Ethiopia, a country in a sub-region that experiences the second highest food borne disease burden in the world, it is difficult to evaluate the burden of food-borne diseases, because of the limited scope of studies and lack of coordinated epidemiological surveillance systems [18].

However, little is known about the prevalence of E. coli serogroup in Ethiopia, either in humans or in the animal population or in foods [37]. Some studies have been conducted in Ethiopia in order to determine the proportion, and antimicrobial susceptibility of E. coli O157:H7 in faeces, skin swabs and carcasses swabs of sheep, goat and cattle in different cities of the country including Addis Ababa 10.2\% [5], Adama and Modjo 4.2\% [16], Dire Dawa 15.89\% [21], and Mekelle 5\% [3]. A report by [5] indicated that E. coli
O157:H7 from human stool and minced beef samples with the rate $5.7 \%$ and $4.9 \%$ respectively in Adama and Addis Ababa Ethiopia. [16] isolated E. coli O157:H7 from beef, sheep meat and goat meat with the prevalence, $8 \%, 2.5 \%$, and $2 \%$ respectively.

\subsection{Significance of the Study}

There is no previously conducted research to isolate, identify appraise associated risk factor and assess antimicrobial susceptible pattern in western parts of Ethiopia in general and in Jimma area in particular. The present study will address the lack of information pertaining to the isolation, identification, associated risk factors and antimicrobial susceptibility pattern of E. coli $\mathrm{O} 157: \mathrm{H} 7$ in raw meat obtained from cattle slaughtered in abattoir and meat from butcher shop in Jimma town. The information from this study helps to build up practical recommendation and guideline of possible ways of controlling contamination caused by Escherichia coli and limit development of drug resistance.

Thus, the current study is designed to achieve the following objective.

General objective

To assess the carcass contamination with strains of Enterohaemorrhagic E. coli O157:H7 in cattle slaughtered at municipal abattoirs and meat of the butcher shop.

Specific objectives

To isolate and identify E. coli O157:H7 from cattle meat at slaughter house and butcher shop.

To assess the antimicrobial susceptibility pattern of E. coli O157:H7 isolates

To appraise risk factor of E. coli O157:H7 at slaughter house and butcher shop.

\section{Materials and Methods}

\subsection{Study Area}

This study was conducted at Jimma municipal Abattoir and Butcher shop in Jimma town between January 2018 and September 2018. Jimma zone is one of the zone in Oromiaregional state which located at $353 \mathrm{~km}$ southwest Finfinne the capital city of the Federal Democratic Republic of Ethiopia. The town is geographically located at $7^{\circ} 41^{\prime} \mathrm{N}$ latitude, $36^{\circ} 50^{\prime} \mathrm{E}$ longitude, and an average altitude of 1,780 meters above sea level and commonly characterized by warm weather with mean annual maximum and minimum temperature of $30^{\circ} \mathrm{C}$ and $14^{\circ} \mathrm{C}$, respectively. The annual rainfall ranges between 1138 and 1690 millimeters [2].

\subsection{Study Design and Sample Size Determination}

A cross-sectional study design was conducted to identify, isolate, appraise associated risk factors and assess the antimicrobial susceptibility pattern of E. coli O157:H7 from meat and different utensil at slaughter house, and meat presented for sale and utensil in different butcher shop. Purposive sampling method was employed on meat and utensil at both slaughtered house and butcher shop, i.e. the 
areas showing contamination with dust or fecal were chosen. In the study period 288 samples were collected from meat and utensil, area showing contamination with fecal or dust. Consequently, 72 meat samples and 72 utensil samples from butcher shops, and 72 carcass samples and 72 utensil samples at slaughter houses were collected.

\subsection{Sample Collection}

Samples from the purposefully selected carcasses and utensil was swabbed as described in ISO17604 (2005) by placing sterile template $(10 \times 10 \mathrm{~cm})$ on specific sites of a carcass. A sterile cotton tipped swab, $(2 \times 3 \mathrm{~cm})$ fitted with shaft, were first soaked in an approximately $10 \mathrm{ml}$ of buffered peptone water (Oxoid Ltd., Hampshire, England) rubbed first horizontally and then vertically several times on the carcasses. The abdomen (flank), thorax (lateral) and breast (lateral.) From utensil such as knife, cutting board swab and butcher man's hand at the shop the same swabbing procedure was followed. On completion of the rubbing process, the shafts were broken by pressing it against the inner wall of the test tube and dispose leaving the cotton swab in the test tube. $25 \mathrm{~g}$ of whole cuts of raw meat sample was collected from butcher shops following aseptic techniques. The samples were put in a sterile universal bottle filled with $225 \mathrm{ml}$ of buffered peptone water. Finally, the samples was transported to the Jimma University College of Agriculture and Veterinary medicine school of veterinary medicine microbiology laboratory using ice box in cold chain for microbiological analysis. Up on arrival, the samples were stored in refrigerator at $4^{\circ} \mathrm{C}$ for $24 \mathrm{hrs}$ until being processed for isolation.

\subsection{Sample Preparation}

Each carcass swabs was homogenized with vortex mixer and $25 \mathrm{~g}$ of raw whole cuts of meat sample collected from butcher shops is chopped aseptically and the meat is placed with $225 \mathrm{ml}$ of buffered peptone water in a plastic bag and homogenized using a homogenizer (Stomacher 400, Seward Medical, England) at high speed for 2 minutes. The resulting suspension is used for isolation of E. coli $\mathrm{O} 157: \mathrm{H} 7$.

\subsection{Isolation and Identification of Escherichia Coli O157:H7}

Isolation and identification of $\mathrm{E}$. coli $\mathrm{O} 157: \mathrm{H} 7$ was performed by standard bacteriological methods. Ninety $\mathrm{ml}$ of modified tryptic soya broth supplemented with novobiocin $(\mathrm{mTSB}+\mathrm{N}$;) was added to $10 \mathrm{ml}$ swab sample. Conversely, $25 \mathrm{gm}$ of meat sample were collected in a Stomacher bag. After adding $225 \mathrm{ml} \mathrm{mTSB}+\mathrm{N}$, each sample was homogenized using a Stomacher 400 (Seward Medical, England) for two minutes and transferred into a sterile flask.

Then, the samples were incubated at $37^{\circ} \mathrm{C}$ for $24 \mathrm{hrs}$ and streaked on Mac Conkey agar (Hamidia Ltd., Cambridge, UK) which is selective and differential medium for E. coli (Hamisi et al., 2012). Then, pink colonies were picked and transferred on to Eosin Methylene Blue (EMB) agar (Hamidia Ltd., Cambridge, UK). Colonies with metallic green sheen on EMB (characteristic of E. coli) were suspected as E. coli (Annex- IV).

Then, the bacterium that was suspected as E. coli on EMB were sub cultured on Sorbitol MacConkey agar (HAMIDIA Ltd., Cambridge, UK) (Annex-I) and were incubated at $37^{\circ} \mathrm{C}$ for 24 hours. The Non-Sorbitol-Fermenting (NSF) E. coli (colorless or pale colonies) was considered as E. coli O157:H7 whereas pinkish colored colonies (Sorbitol-Fermenters) was considered as non O157:H7 E. coli (Annex- IV). Colonies with colorless or pale colonies (characteristic of E. coli) were taken to different biochemical test (Annex- IV). After isolation of the organism on the selective media, triple sugar iron (TSI) agar (Difco, MI, USA) was used for further characterization. Yellow slant, yellow butt, presence of gas bubbles, and absence of black precipitate in the butt which indicates E. coli (Islam et al., 2014) subsequently, the isolates was subjected to different biochemical tests according to Quinn et al. (2002) such as indole production test, motility test, and citrate utilization (IMViC) test (Annex- IV).

The sample that shows Escherichia coli character on these all biochemical test was taken to NAHDIC for further phonotypical characterization by transported media $20 \%$ glyceriline with tryptic soya broth to analyze using BIOLOG identification system (BiOLOG User Guide, 2008).

The transported sample was streaked on Brain heart infusion and pure colonies from Brain heart infusion agar were inoculated on Biolog Universal Growth Medium (BUG) agar (Hayward, USA) with 5\% sheep blood and incubated at $37^{\circ} \mathrm{C}$ for $24 \mathrm{hrs}$. Sub-culture was made using the same culture media to have pure culture colonies before identification was done by OmniLog (Annex-I). The BUG (Biolog Universal Growth Medium) is a recommended medium for aerobic bacteria and it was employed to isolate E. coli. Following this, the identification of Escherichia coli O157:H7 was performed using the Omnilog plus Identification System (BiOLOG User Guide, 2008).

After getting pure culture colonies, identification was carried out using BiOLOG Standard Operation Protocols (SOP). For each inocula preparation, the turbidometer was blank with the 91-98 inoculated inoculating fluid tube (wiped clean of dirt and fingerprints) by adjusting the $100 \%$ transmittance adjustment knob so that the meter reads $100 \%$ transmittance (i.e. Inoculating Fluid and Cell Density). For aerobic, enteric bacteria identification, having 91-98\% turbidity in 18-20 ml inoculating fluid was adjusted (AnnexIV). A sterile cotton swab was used and the top of a colony was gently touched to pick up a $3 \mathrm{~mm}$ diameter area of cell growth from the surface of the agar plate and the organisms were emulsified into the solution using a vigorous motion on the bottom of the inoculating fluid tube to release the bacteria into the inoculating fluid. Any bubble that may have formed was allowed to disperse. The turbidity was read using turbidimeter (annex, I).

The $91-98 \%$ turbidity suspension was adjusted by adding more cells (to increase density) or more inoculating fluid (to lower density). The Micro Plate was labeled to the side of the Micro Plate itself, not the lid (Biolog imprint) side. Using aseptic technique, the cell suspension was poured into the 8- 
Channel repeating pipette (Annex I). As shown (Annex- IV) all Micro Plate wells were filled with 100 ( $\mu \mathrm{L} /$ well) by avoiding contamination and touching the bottom of the wells, which could transfer carbon sources then the Micro Plate was covered with its lid. The plates were placed in OmniLog machine (Annex- IV) at $33^{\circ} \mathrm{C}$ for $22 \mathrm{hrs}$ (BiOLOG User Gide, 2008; (Annex- IV). After incubation time, OmniLog Identification System automatically read each Micro Plate (Annex- IV). The patterns formed from the wells were automatically entered to the system and used for identification (BiOLOG User Gide, 2008).

\subsection{Antimicrobial Susceptibility Test}

The antimicrobial susceptibility test was performed following the standard agar diskdiffusion method according to CLSI (Wayne, 2012) using commercially available antimicrobialdisks. Each isolated bacterial colony from pure fresh culture was transferredinto a test tube of $5 \mathrm{ml}$ Tryptone Soya Broth (TSB) (Oxoid, England) and incubated at $37^{\circ} \mathrm{C}$ for $6 \mathrm{hrs}$. The turbidity of the culture broth was adjusted using sterile saline solutionand by adding more isolated colonies to obtain turbidity usually comparable with that of $0.5 \mathrm{McF}$ arland standards (approximately $3 \times 108 \mathrm{CFU}$ per $\mathrm{ml}$ ). Mueller-Hinton agar (Bacton Dickinson and Company, Cockeysville USA) plates were prepared according tothe manufacturer guidelines. A sterile cotton swab was immersed into the suspension androtated against the side of the tube to remove the excess fluid and then swabbed in threedirections uniformly on the surface of Mueller-Hinton agar plates. After the plates dried, antibiotic disks were placed on the inoculated plates using sterile forceps. The antibioticdisks were gently pressed onto the agar to ensure firm contact with the agar surface, andincubated at $37^{\circ} \mathrm{C}$ for $24 \mathrm{hrs}$. Following this the diameter of inhibition zone formedaround each disk was measured using a black surface, reflected light and transparent rulerby lying it over the plates. The results were classified as sensitive, intermediatelyresistant, and resistant according to the standardized table supplied by the manufacturer (CLSIFAD, 2012).

\subsection{Data Management and Analysis}

In order to have the desired analysis, the coded data was entered in to MS-Excel and then analyzed using SPSS version 20. Descriptive statistics (determination of proportions) was used to summarize the generated data on the rate of E. coli O157:H7 enterohaemorrhagicE. coli isolation, associated risk factor and antibiotic susceptibility pattern of the E. coli O157:H7 isolates. The proportion of positive was calculated by the number of positive samples divided by the total number of samples examined and multiplied by 100 . Difference among and between proportions of the groups with certain determinant factors were determined by fisher exact test. A pvalue $<0.05$ was considered to be statistical significant.

\subsection{Ethical Consideration}

Before conducting this research, all the owners of different butcher house and abattoir workerwere informed about the purpose of the study and also them well aware of the importance and benefit of the research in terms of immediate and future values. Besides, the research was highly participatory in the sense that different catering establishment's owner was provided their hotels/restaurants/retail shops as research grounds.

\section{Results}

\subsection{Prevalence of Escherichia Coli at Slaughter House and Butcher Shop}

Of the total 288 samples examined, 51 were positive for E. coli using differential and selective culture media Mac konkey, EMB, SMAC and different biochemical tests. From all these 51 suspected E. coli characterized by Biolog four of them are confirmed phenotypically as E. coli O157:H7 strain in which one from butcher shop and other three were from slaughter houses when disaggregated according to the types of samples examined, the specimens positive for E. coli O157:H7 were: carcass swab (2) $(2.77 \%)$ at slaughter house, meat at butcher shop (1) (1.38\%), utensil at slaughter house (1.38) and zero prevalence of utensil at butcher shop (Table 1).

Table 1. Prevalence of Escherichiacoli O157:H7 at different sample sources.

\begin{tabular}{lllll}
\hline Sample source & Sample type & $\begin{array}{l}\text { Number of sample } \\
\text { examine }\end{array}$ & $\begin{array}{l}\text { Phenotypically charecterized } \\
\text { e. coli O157:H7 }\end{array}$ & $\begin{array}{l}\text { Phenotypically charecterized e. } \\
\text { coli O157:H7\% }\end{array}$ \\
\hline \multirow{3}{*}{ Abattoir } & Carcass swab & 72 & 2 & $2.77 \%$ \\
& Knife swab & 24 & 0 & $0 \%$ \\
& Personnel hand swap & 24 & 0 & $0 \%$ \\
& Meat hanging up wire swab & 24 & 1 & $4.16 \%$ \\
Butcher shop & Meat & 72 & 1 & 1.38 \\
& cutting board swab & 24 & 0 & $0 \%$ \\
Total & Personnel hands swab & 24 & 0 & $0 \%$ \\
\hline
\end{tabular}

\subsection{Antimicrobial Susceptibility Pattern}

The result of antimicrobial susceptibility test of 4 E. coli O157:H7 isolated from carcass swab slaughter house and meat at butcher shop as well as one from meat hanging up wire swab different subjected to 12 selected antimicrobial agents are shown in Table 2. The current study on antimicrobial susceptibility test of E. coli O157:H7 recovered from different sample types revealed a varying degree of susceptibility to antimicrobial agents tested. 
Escherichia coli O157:H7 was highly susceptible to Kanamycin (100\%), Chloramphenicol (100\%), Ciprofloxacin $(100 \%)$, Streptomycin $(100 \%)$, tetraccine $(100 \%)$, Gentamicine (100\%), Trimethoprim (75\%), \&Sulfamide (75\%). Intermediate susceptibility was also observed in Trimethoprim (25\%),
Sulfamide (25\%), Neomycin (25\%), Oxytetracycline (75\%) and Cefoxitin (50\%). Furthermore, resistance of 100\%, 75\% 50\% and $25 \%$ was developed to ampicillin, neomycin, cefoxitin and oxytetracycline respectively.

Table 2. Susceptibility range (resistant, intermediate and susceptible) in each antimicrobial agent for each isolates.

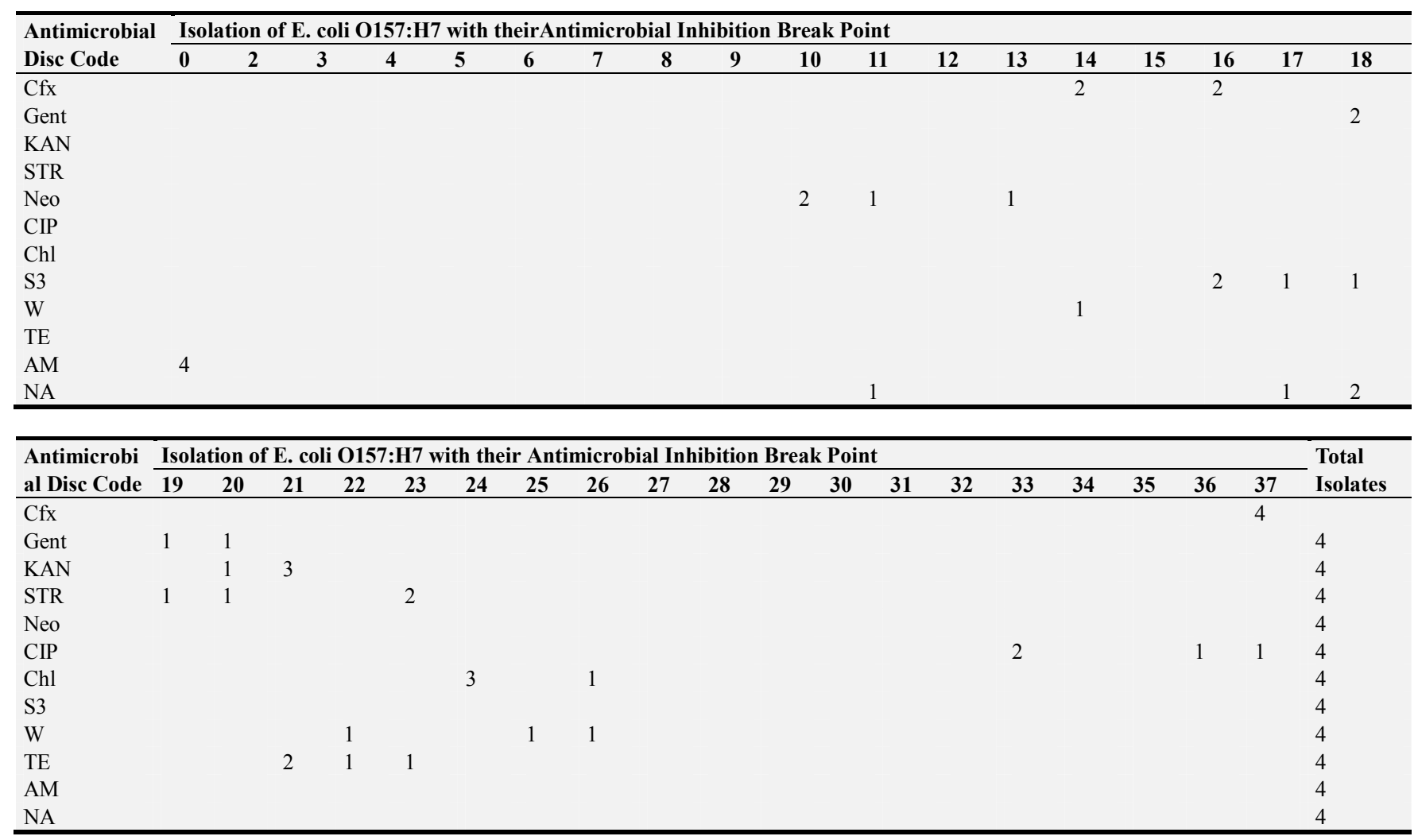

Foot notes: - Cfx, cefoxitin, gent, Gentamicine, KAN: Kanamicine, STR: Stryptomycine, Neo: Neomycine, Chl: Chloromphenicol, S3: sulfonamides, W: Trimethophorim, TE: Tetracycline, AM: Ampicilline, NA: Oxytetracycline

Note: the line shows the diameter of zone of inhibition. The number to the left side of break point shows bacteria that resistance to the antimicrobial and number between the break point lines shows intermediate to antimicrobial were as the number right side of break point represent susceptibility of microbial to antimicrobial.

The antimicrobial susceptibility profile showed a significant difference $(\mathrm{p}<0.05)$ in the susceptibility pattern of various antimicrobial agents among E. coli O157:H7 isolates recovered from different meat and utensil at butcher shop and slaughter houses. Isolates recovered from carcass swab at slaughter house were found to be 16.66 to $33.33 \%$ resistant in carcass swab and $50 \%$ to $66.66 \%$ susceptible in at slaughter house. In butcher shop $66.66 \%$ susceptible, $16.66 \%$ intermediate and $16.66 \%$ resistance to the tested antibiotics.

Table 3. Antimicrobial susceptibility test result of Escherichia coli O157:H7 isolates by percent.

\begin{tabular}{lllll}
\hline \multirow{2}{*}{ Antimicrobial agent } & \multirow{2}{*}{ Disk concentration } & \multicolumn{2}{l}{ Susceptible intermediate and Resistance pattern of Escherichia coli O157:H7 isolates } \\
\cline { 3 - 5 } & & S\% & R\% & I\% \\
\hline KA & $30 \mu \mathrm{g}$ & $4(100 \%)$ & 0 & 0 \\
W-5 & $5 \mu \mathrm{g}$ & $3(75 \%)$ & 0 & 0 \\
CHL & $30 \mu \mathrm{g}$ & $4(100 \%)$ & 0 & 0 \\
CPR & $5 \mu \mathrm{g}$ & $4(100 \%)$ & 0 & 0 \\
STR & $10 \mu \mathrm{g}$ & $4(100 \%)$ & 0 & 0 \\
TTC & $30 \mu \mathrm{g}$ & $4(100 \%)$ & 0 & 0 \\
SLF & $0.3 \mu \mathrm{g}$ & $4(100 \%)$ & 0 & 0 \\
Gent & $10 \mu \mathrm{g}$ & $0(100 \%)$ & 0 & $1(25 \%)$ \\
Neo & $30 \mu \mathrm{g}$ & 0 & $3(75 \%)$ & $2(50 \%)$ \\
Amp & $10 \mu \mathrm{g}$ & 0 & $2(100 \%)$ & $(75 \%)$ \\
Cfx & $30 \mu \mathrm{g}$ & & $1(25 \%)$ & 0 \\
NA & $30 \mu \mathrm{g}$ & & & \\
\hline
\end{tabular}




\subsection{Risk Factors of E. coli O157:H7 at Butcher Shops and Slaughter House}

Once the overall prevalence of E. coli was determined, its prevalence related to multiple risk factors was also assessed as described in tables $4 \& 5$ in which both washing cutting board, butcher shop cleaning and carcass contact to fecal, rumen content removal method, meat transportation method, and carcass contact to skin at slaughter house were found to be have a significant difference on the prevalence of E. coli $(\mathrm{P}<0.05)$ and where as other of risk factors did not show any significant difference $(\mathrm{P}>0.05) 4 \& 5$.

Table 4. Risk factor at slaughter houses.

\begin{tabular}{|c|c|c|c|c|}
\hline Variable & Frequency (\%) & $\mathrm{X}^{2}$ & Df & p-value \\
\hline Hands and knife wash & & 3.50 & 1 & \\
\hline Yes & $112(77.8 \%)$ & & & 1.24 \\
\hline No & $32(22.2 \%)$ & & & \\
\hline Water source at slaughter house & & 0.204 & 1 & \\
\hline Pond & $135(93.8 \%)$ & & & 0.823 \\
\hline Flow water & $9(6.2 \%)$ & & & \\
\hline Wear gown & & 0.41 & 1 & \\
\hline Yes & $127(88.2 \%)$ & & & 0.64 \\
\hline No & $17(11.8 \%)$ & & & \\
\hline Carcass contact to fecal & & 36.5 & 1 & \\
\hline Yes & $5(3.5 \%)$ & & & 0.003 \\
\hline No & $139(96.5 \%)$ & & & \\
\hline Rumen content removal & & 29.97 & 1 & \\
\hline Opened in house & $6(4.2 \%)$ & & & 0.004 \\
\hline Not opened in house & $138(95.8 \%)$ & & & \\
\hline Carcass can contact to skin & & 36.50 & 1 & \\
\hline Yes & $5(3.5 \%)$ & & & 0.003 \\
\hline No & $139(96.5 \%)$ & & & \\
\hline Meat transportation method & & 21.80 & 1 & \\
\hline Mixed with rumen content & $8(5.6 \%)$ & & & 0.008 \\
\hline Red meat is separated & $136(94.4 \%)$ & & & \\
\hline Knife used to cut meat and rumen & & 4.95 & 1 & \\
\hline The same knife & $55(38.2 \%)$ & & & 0.054 \\
\hline Not the same & $89(61.8 \%)$ & & & \\
\hline Escherichia coli status at slaughter house & & & & \\
\hline Positive & $3(2.1 \%)$ & & & \\
\hline Negative & $141(97.9 \%)$ & & & \\
\hline
\end{tabular}

Table 5. Risk factor at butcher shop.

\begin{tabular}{|c|c|c|c|c|}
\hline Variable & Frequency (\%) & $\mathbf{X}^{2}$ & df & p-value \\
\hline Washing cutting board & & 1.440 & 1 & \\
\hline yes & $143(99.3)$ & & & 0.007 \\
\hline no & $1(0.7)$ & & & \\
\hline Use disinfectant to cutting board & & 0.029 & 1 & \\
\hline Yes & $140(97.2 \%)$ & & & 0.972 \\
\hline No & $4(2.8 \%)$ & & & \\
\hline Butcher shop cleaning & & 1.440 & 1 & \\
\hline Yes & $142(98.6 \%)$ & & & 0.007 \\
\hline No & $2(1.6 \%)$ & & & \\
\hline Butcher shop is forwarded to sun light & & 0.044 & 1 & \\
\hline Yes & $138(95.8 \%)$ & & & 0.958 \\
\hline No & $6(4.2)$ & & & \\
\hline covering meat by plastic & & 0.051 & 1 & \\
\hline Yes & $137(95.1 \%)$ & & & 0.951 \\
\hline No & $7(4.9 \%)$ & & & \\
\hline Escherichia coli status at butcher shop & & - & - & \\
\hline Positive & $1(0.7 \%)$ & & & - \\
\hline Negative & $143(99.3 \%)$ & & & \\
\hline
\end{tabular}

Foot note: $\mathrm{X}^{2}$ : fisher exact test

\section{Discussion}

Food borne diseases is one of the important issues in the $21^{\text {st }}$ century all over the world. Escherichia coli $\mathrm{O} 157: \mathrm{H} 7$ is considered the most prevalent food borne pathogen that has gained increased attention worldwide in recent years. The present study was conducted to establish isolation, identification and antimicrobial susceptibility pattern of E. 
coli $\mathrm{O} 157: \mathrm{H} 7$ as well as associated risk factor on samples collected from abattoir and butcher shop in Jimma town.

At butcher shop level the prevalence of E. coli O157:H7 was $1.38 \%(1 / 72)$ from meat and $0 \%$ prevalence from utensil. This result is in line with $1.03 \%$ from Ethiopia [40] $1.2 \%$ prevalence in USA [3], 1.4\% in England [8], 1.5\% from Turkey [13] and 1.6\% from Canada [27]. But this finding is lower than some reports from previous works: $5.5 \%$ from Ethiopia [21] and 8\% [16], 3\% from Ireland [7], 6.4\% from Isfahan [28], 8.3\% from Iran [15] and 9.6\% from Iran [36]. In contrast the finding of the present study is a bit higher than prevalence of $0.4 \%$ from France $[14,17]$.

Observed variation in prevalence among studies could be attributed to difference in sampling and isolation procedures, fecal and skin contact to carcass, method of meat transportation to butcher house, method of rumen content removal, abattoir conditions, study design, season and treatment with antimicrobial substances during the process (disinfectants). A number of studies have confirmed that the prevalence of E. coli O157:H7 varies among studies due to the above mentioned reasons [8].

In this study, E. coli O157:H7 was not isolated from utensil at butcher shop. In a similar kind of study conducted in Pakistan, E. coli O157:H7 was not detected in surface swabs (knives, cutting boards, weighing scales, and meat mincers) taken from 30 individual retail meat outlet markets [15]. This may be due to the butcher man consideration about food born microorganism and safety taken for cleaning in butcher shops.

Even though, the highest prevalence was observed in carcass swab sample 2 from $72(2.77 \%)$ among the different sample types of slaughterhouse and $1(1.388 \%)$ from utensil at slaughter house. Similar findings were reported by [22] from United Kingdom (2.4\%) and [9] in Irish abattoir (3\%). Conversely, our finding is lower than the reports by $[35,25]$ in United Kingdom (8.6\%), [24] in United Kingdom (7.5\%), [6] in United State (11.3\%), [26] in United Kingdom (40.4\%), [10] in United State 28\%, [35] in United Kingdom 23.7\%.

The differences in the reported prevalence could be due to the used culture media, condition of slaughtered house, cleaning method and season in which sample is collected [41]. The greater prevalence encountered in the present study as compared with the previous once may be due to the presence of sorbitol mackonkey (SMAK) technique of isolation methods. Several enrichment culturing methods and isolation methods have been developed but SMAK technique is the most sensitive for O157:H7 strain. [39]. on the other hand the present study prevalence lower than the previous mentioned at the above paragraph it might be due to seasonal variation. The present sample was collected during the winter season. Seasonal distribution of Escherichia coli O157:H7 has been reported previously by [7] [38] with highest prevalence in summer and lowest in winter so it is possible that the contamination rate lower than previous studies.

In the present study, all of the $4 \mathrm{E}$. coli O157:H7 isolates were highly susceptible to Tetracycline (TE30 $\mu \mathrm{g}$ ), Kanamycin, (K30 $\mu \mathrm{g})$, trimethoprim (SXT25 $\mu \mathrm{g})$, Ciprofloxacin (CIP5 $\mu \mathrm{g})$ and Chloramphenicol (C30 $\mu \mathrm{g})$. This finding agrees with the work of $[16,33]$.

However, the study conducted in Saudi Arabia (Naser and Wabel, 2007), revealed that there was resistant strain to the drugs such as Tetracycline (TE30 $\mu \mathrm{g})$, Kanamycine $(\mathrm{K} 30 \mu \mathrm{g})$,

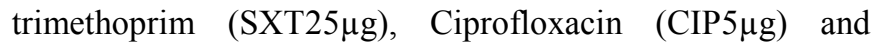
Chloramphenicol $(\mathrm{C} 30 \mu \mathrm{g})$. This variation probably attributed to the expression of resistant gene code by the pathogen which associated with emerging and re-emerging aspects of the isolates with the regards of different agro ecology (Reuben and Owuna, 2013). On the other side, the current study revealed that all isolates were $75 \%$ resistant to Neomycin (AML25 $\mu$ g). Similar findings were reported by many researchers [23]. This might be due to the use of inappropriate antibiotics for treatment of diseases [34] and also excessive use of antimicrobials for therapeutic and prophylactic treatment $[20,31]$.

One isolate have multiple drug resistance in our findings. This result comparable related with previous findings [41]. This multi-drug resistance occurrence might be due to administration of multiple antibiotics for prophylaxis or infection, discriminate use of antibiotics in the farms and another possibility is that cattle are being treated with antibiotics for other conditions, there by selecting for resistant populations of E. coli O157:H7. Such multi drug resistance may apparently be occurred which may ultimately replace the antimicrobial sensitive microorganisms from antibiotic saturated environment [35].

Association of several risk factors with carcass contaminations has been reported by several researchers at slaughter house and butcher shops. Associations with feces ([10] and skin [30] and has been reported where E. coli O157:H7 can spread easily on to carcass surfaces from the hide or during evisceration [10]. The result of the present study was therefore in agreement with the previous studies.

According to the report by [4], the infective dose of the pathogen is $<10$ cells for humans. Considering this very low infective dose of this pathogen, its detection in the butcher houses and abattoir of this study poses public health risks.

\section{Conclusion and Recommendation}

Escherichia coli $\mathrm{O} 157: \mathrm{H} 7$ is considered the most prevalent food borne pathogen that has gained increased attention worldwide in recent years. The present study showed an important presence of E. coli O157:H7 strain in cattle meat slaughtered at abattoir and meat from butcher shop in the town. In this study, the overall prevalence of E. coli O157:H7 that contaminate the meat of cattle at slaughter house was higher that of the butcher shop. The perceive taken for safety at butcher shop well thought-out practice could be sensible continued and the same practice could allowed in slaughtered houses furthermore. Escherichia coli O157:H7 was isolated from meat at butcher shop this indicate that as bacteria infective dose was very miniature it is perilous problem on human health.

The occurrence of E. coli had significant difference with washing cutting board and cleaning method at butcher shop, 
where as carcass contact to feces and skin, rumen content removal method and meat transportation method were associated risk factor at slaughter houses.

Though most of the E. coli isolates subjected to antimicrobial susceptibility tests show different degrees of resistance against the antimicrobial discs tested, none of them were found to be susceptible to Ampicilin. The isolated bacteria were susceptible to most of the drugs used and multi drug resistance was occurred in one isolate for in vitro testing in this study.

Based on the above concluding remarks, the following recommendations are forwarded:

City administration should bestow rigorous trainings to those personnel working in municipal slaughter houses to ensure the hygienic practices during slaughtering of animals on cleaning dirty before animals slaughtering, skinning while being on the rail, separating carcasses from each other and avoiding contact between the external surface of the hide and carcasses.

Hygiene measures must be sufficient to prevent from contamination via hands, knives, saws, equipment, clothing and regular medical checkup of personnel working in both abattoir and butcher houses.

In vitro drug sensitivity testing of E. coli O157:H7 should be performed so that proper treatments can be instituted for E. coli $\mathrm{O} 157: \mathrm{H} 7$ infected patients at animal and human health center.

Microbial load must be done by responsible body.

Further molecular characterization of both E. coli O157:H7 and other shiga toxin producing E. coli strains should be conducted.

\section{Acknowledgements}

First and for most, I would like to address my genuine thanks to my major advisor, Prof. TadeleTolosa and co adviser Dr. MukarimAbdurahaman for their patience, motivation, and immense knowledge in continuous support of my Msc study and research.

It is also my pleasure to thank Jimma University for giving an opportunity to pursue my Msc study and financial support for research work.

My sincere thanks also goes to National Animal Health Diagnostic and Investigating Center NAHDIC Dr. Matios Lakew and Mr. AbebeSagni for giving me an opportunity to join their team as an intern, and access to the laboratory and research facilities.

I thank my lab mates DiribaTadese for the sleepless nights we were working together before deadlines, and for all the fun we have had together in the last two years.

I am also grateful to Jimma Abattoir and all Jimma meat retail shops for their willingness during sample collection.

Last but not the least, I would like to thanks my wife, my parents and to my brothers and sister for supporting me spiritually throughout writing this thesis and my life in general.

\section{References}

[1] Akond, M. A., Alam, S., Hasan, S. M., Mubassara, S., Uddin, S. N. and Shirin, M. (2009): Antibiotic resistance of Escherichia coli isolated from poultry and poultry environmentof Bangladesh. Am. EnvSci 5: 47-52.

[2] Alemu, W. Tsegaye, L. Golassa, and G. Abebe, (2011): "Urban malaria and associated risk factors in Jimma town, south-west Ethiopia," Malaria J. 10: 173-200.

[3] Barkocy-Gallagher, G. A., Arthur, T. M., Rivera-Betancourt, M., Nou, X., Shackelford, S. D., Wheeler, T. L and Koohmaraie, M. (2003): Seasonal prevalence of Shiga toxin producin Escherichia coli, including O157:H7 and non-O157 serotypes, and Salmonella incommercial beef processing plants. J Food Prot, 66: 1978-1986.

[4] Bassam, Y., Khudaier, B., Abbas, A. and Khulood, A. (2012): Prevalence and antimicrobialsusceptibility of E. coli O157:H7 isolated from human and animal sources in Basrahprovince. J. Vet. Res., 11: 2 .

[5] Beyi, A. F., Fite, A. T., Tora, E., Tafese, A., Genu, T., Kaba, T., ... Cox, E. (2017): Prevalence and antimicrobial susceptibility of Escherichia coli O157 in beef at butcher shops and restaurants in central Ethiopia. BMC Microbiol, 17, 49.

[6] Callaway, T. R., Carr, M. A., Edrington, T. S., Anderson, R. C., Nisbet, D. J. (2009): Diet, Escherichia coli O157:H7, and cattle: a review after 10 years. CurrIssuesMolBiol, 11: 67-79.

[7] Carney, E., O’Brien, S. B., Sheridan, J. J., Mcdowell, D. A., B lair I, S., Duffy, G. (2006): Prevalence and level of Escherichia coli $\mathrm{O} 157$ on beef trimmings, carcasses and bonedhead meat at a beef slaughter plant. Food Microbiol, 23: 52-59.

[8] Chapman, P. A., Cerdán, A. T, Ellin, M., Ashton, R., Harkin, M. A. (2001): Escherichia coli O157 in cattle and sheep at slaughter, on beef and lamb carcasses and in raw beef and lambproducts in South Yorkshire, UK. Int J Food Microbiol, 64: 139-150.

[9] Conedera, G., Dalvit, P., Martini, M., Galiero, G., Gramaglia, M., Goffredo, E., Loffredo, G., Morabito, S., Ottaviani, D., Paterlini, F., Pezzotti, G., Pisanu, M., Semprini, P. andCaprioli, A. (2004): Verocytotoxin producing escherchia. coli $\mathrm{O} 157$ in minced beefand dairy product in Italy. Int. J. Food Microbiol, 96: 67-73.

[10] Elder, R., Keen, J., Siragusa, G., Barkocy, G., Koohmaraie, M. and Laegreid, W. (2000): Correlation of Enterohemorrhagic Escherichia coli $\mathrm{O} 157$ prevalence in faeces, hides andcarcasses of beef cattle during processing. ProcNatlAcadSci USA, 97: 2999-3003.

[11] Frye JG and Jackson CR (2013): Genetic mechanisms of antimicrobial resistance identified in Salmonella enterica, Escherichia coli, and Enterococcus Spp. Isolated from U.S. food animals. Front Microbiol.; 4: 135.

[12] Gansheroff LJ, O’Brien AD (2000): Escherichia coli O157:H7 in beef cattle presented for slaughter in the US: Higher prevalence rates than previously estimated. ProcNatlAcadSci USA. 97: 2959-2961.

[13] Guerra, B., Junker, E., Schroeter, A., Malorny, B., Lehmann, S. and Helmuth, R. (2003): Phenotypic and genotypic characterization of antimicrobial resistance in GermanEscherichia coli isolates from cattle, swine and poultry. J. Anti. Chem, 5: 489-92. 
[14] Guyon, R., Dorey, F., Malas, J. P., Grimont, F, Foret, J., Rouvière, B., Collobert, J. F. (2001): Superficial contamination of bovine carcasses by Escherichia coli O157:H7 in slaughterhouse in Normandy (France). Meat Sci, 58: 329-331.

[15] Hassan Ali N, Farooqui A, Khan A, Khan AY, Kazmi SU (2010). Microbial contamination of raw meat and its environment in retail shops in Karachi, Pakistan. J Infect DevCtries.; 4: 3828.

[16] Hiko, A., Asrat, D. and Zewde, G. (2008): Occurrence of Escherichia coli $\mathrm{O} 157: \mathrm{H} 7$ in retail rawmeat products in Ethiopia. J Infect Dev Ctries. 2: 389-393.

[17] ISO 16654: 2001. $1^{\text {st }}$ ed. Microbiology-Horizontal method for the detection of Escherichia coli o157, international organization for Standardization, Geneva, Switzerland.

[18] Kebede, Jelalu., H A., and Solomon H M., (2016): Isolation, Identification, and Antibiotic Susceptibility Testing of Salmonella from Slaughtered Bovines and Ovines in Addis Ababa Abattoir Enterprise, Ethiopia: Int. J. Bact. 2016, 8.

[19] Lindsey RL, Frye SN, Thitaram RJ, Meinersmann PJ. Fedorka-Cray, Englen MD. (2011): Characterization of multidrug-resistant Escherichia coli by antimicrobial resistance profiles, plasmid replicon typing, and pulsed-field gel electrophoresis. Microb Drug Resist. 17: 157-63.

[20] Majalija, S., Francis, O., Sarah, W. G., Lubowa, M., Vudriko, P. and Nakamya, F. M. n (2010). Antibiotics susceptibility profiles of fecal Escherichia coli isolates from Dip Litterbroilers chickens in Northern and Central Uganda Department of veterinary parasitologyand microbiology. Veterinary Research 3: 75-80.

[21] Messele, Y. E., Abdi, R. D., Yalew, S. T., Tegegne, D. T., Emeru, B. A. and Werid, G. M., 2017. Molecular determination of antimicrobial resistance in Escherichia coli isolated from raw meat in Addis Ababa and Bishoftu, Ethiopia. An cl microbi and antimicrobial, 16. 55.

[22] Molbak K. (2004): Spread of resistant bacteria and resistance genes from animals to humans, the public health consequences. J Vet Med.; 51: 364-9.

[23] Mora, A., Blanco, J. E., Blanco, M., Alonso, M. P. and Dhabi, G. (2005): Antimicrobial Resistanceof Shiga Toxin (Verotoxin)-Producing E. coli O157:H7 and Non-O157 Strains Isolatedfrom Humans, Cattle, Sheep and Food in Spain. Res. Microbiol. 156: 793-806.

[24] Omisakin, F., MacRae, M., Ogden I. D and Strachan., N. J. C (2003): Concentration and Prevalenceof Escherichia coli O157 in Cattle Feces at Slaughter Society for Microbiology UnitedKingdom 5. 2444-47.

[25] Osaili, M., Alaboud, R. and Rahahlah, M. (2013): Prevalence and antimicrobial susceptibility of E. Coli O157:H7 on beef cattle slaughtered in Amman abattoir. Meat. Sci., 93: 463-468.

[26] Pallecchi, L., Bartoloni, A., Fiorelli, C., Mantella, A., Di Maggio, T., Gamboa, H., Gotuzzo, E., Kronvall, G., Paradisi, F. and Rossolini, G. M. (2007): Rapid dissemination and diversity of CTX-M extended-spectrum beta-lactamase genes in commensal Escherichia coli isolates from healthy children from low-resource settings in Latin America. Antimicrob Agents Chemother 51, 2720-2725.

[27] Power, C. A., Johnson, R. P., Mcewen, S. A., McNab, W. B.,
Griffiths, MW., Usborne, W. R., DeGrandis, S. A. (2000). Evaluation of the reversal and Safe Path rapid Escherichia coli O157 detection tests for use on bovine feces and carcasses. J. Food Prot. 63, 860-866.

[28] Rahimi, E., Momtaz, H., Mohammad, M., Hosseini, A., Alimoradi, M., Momeni, Mand. Riahi, M. (2012): Isolation and genomic characterization of Escherichia coli O157: NM and Escherichia coli O157:H7 in minced meat and some traditional dairyproducts in Iran. Afri J Biotech. 11: 2328-2332.

[29] Raji MA, Minga U, Machangu R (2006): Current epidemiological status of enterohaemorrhagic Escherichia coli O157:H7 in Africa. Chin Med J (English) 119: 217-22.

[30] Reed, Jennifer Leanne (2005): "Model Driven Analysis of Escherichia coliMetabolism., 200-5-18.

[31] Reuben, R. and Owuna, G. (2013): Antimicrobial resistance patterns of E. coliO157:H7 fromNigerian fermented milk samples in Nasarawa state, Nigeria. Int. J. Pharma. Sci. Invention., 2: 2319-6718.

[32] Robins-Browne, R. M. (2005): The relentless evolution of pathogenic Escherichia coli. Clin Infect Dis 41, 793-794.

[33] Salehi, T. Z. and Bonab S. F. (2006). Antibiotics susceptibility pattern of Escherichia colistrains isolated from chickens withcolisepticemia in Tabriz Province, Iran. Department of microbiology and immunology, Faculty of Veterinary Medicine, Tehran University. Int. J. Poultry Sci., 5: 677-684.

[34] Sharma S, Bhat GK, Shenoy S (2007): Virulence factors and drug resistance in Escherichia coli isolated from extraintestinal infections. Indian J Med Microbiol., 25: 369-373.

[35] Synge, B. A., Gunn, G. J., Ternent, H. E., Hopkins, G. F., Thomson-Carter F., Foster G., and Mc Kendrick, I. (2000): Preliminary results from epidemiological studies in cattle in Scotland. In Zoonotic infections in livestock and the risk to public health, United Kingdom. 10-17.

[36] Tahamtan, Y. E., Pourbakhsh, S. A. and Shekarforoush, S. S. (2006): PCR detection of Escherchia Coli O157:H7 directed from slaughtered cattle in Shiraz, Iran. Archives Razi institute. 1: 1-6.

[37] Tsegaye M, Ashenafi M. (2005): Fate of Escherichia coli O157:H7 during the processing and storage of Ergo and Ayib, traditional Ethiopian dairy products. Int J Food Microbiol 103: 11-21.

[38] Tutenel, A. V Pierard, D., VanHoof, J, Cornelis, M and De Zutter, L (2003): Isolation andmolecular characterization of Escherichia coli O157 isolated from cattle, pigs and chickens at slaughter. Int J Food Microbiol., 84, 63-69.

[39] Vidal, R., Vidal M., Lagos R., Levine M., Prado V. (2004): Multiplex PCR for the diagnosis of enteric infections associated with diarrheagenicEscherchia coli. J. Clin. Microbiol. 42: 1787-1789.

[40] Wayne (2012): Clinical and Laboratory Standards Institute (CLIS). Performance standards for antimicrobial susceptibility testing; twenty-second informational supplement. M100-S21.

[41] Zhao, S., Maurer J. J., Hubert, S., De Villena, J. F., Mcdermott, P. F., Meng, J., Ayers S., English, L. And White, D. G. (2005). Antimicrobial susceptibility and molecular characterizeation of avian pathogenic Escherichia coli isolates. Vet. Microbiol., 107, 215-24. 